\title{
Öffentlicher Brief
}

Positionspapier der Schweizerischen Akademie für Psychosomatische und Psychosoziale Medizin (SAPPM) und der Schweizerischen Gesellschaft zum Studium des Schmerzes (SGSS) zum BGE-Urteil 9C_492/2014 betreffs somatoformer Schmerzerkrankungen:

1. Aus medizinisch-fachärztlicher Sicht wird der Paradigmenwechsel des Bundesgerichtes mit dem BGE-Urteil 9C_492/2014 vom 03.06.15 ausdrücklich begrüsst. Diese dringende Richtungskorrektur zur Beurteilung somatoformer Schmerzerkrankungen wurde unsererseits lange erwartet.

2. Gemäss Expertise der unterschreibenden Fachleute lehnt sich indessen der daraufhin entworfene Indikatorenkatalog des Bundesamtes für Sozialversicherungen (IV-Rundschreiben Nr. 339 vom 09.09.15) zu einseitig an Qualitätskriterien für psychiatrische Gutachten an und ignoriert die internationalen schmerzmedizinischen Begutachtungsleitlinien. Das im IV-Rundschreiben vorgeschlagene Instrument ist damit fachlich anfechtbar und widerspricht der Richtungskorrektur des Bundesgerichts.

3. Medizinische Fachleute von Schweizer Universitätskliniken sowie der sachzuständigen Fachgesellschaften (SAPPM und SGSS) sind bereit, an der Entwicklung einer medizinisch fundierten zeitgemässen Begutachtung für die Schweiz mitzuarbeiten und damit der Aufforderung des Bundesgerichtes nach Integration von mehr Fachexpertise im modernen medizinischen Begutachtungswesen zu entsprechen.

4. Ebenfalls sind Arbeitsgruppen der zuständigen Fachgesellschaften grundsätzlich bereit, an innovativen und kosteneffektiveren versicherungsmedizinischen Modellen im Hinblick auf die Zunahme von Stress- und Schmerzerkrankungen mitzuarbeiten. 
Dr. med.

Alexander Minzer

Präsident SAPPM

Rothrist

Dr. med.

André Ljutow

Präsident SGSS

Leiter Zentrum für Schmerzmedizin

Nottwil

Dr. med.

Pierre Loeb

Past President SAPPM

Präsident wissenschaftl. Beirat SAPPM,

Basel

PD Dr. phil.

Christine Cedraschi

Past President SGSS

Service de Médecine

Interne de Réhabiliation

Beau-Séjour, HUG

Genève

Prof. Dr. med.

Anne-Françoise Allaz

Médecin chef du Service de Médecine interne de réhabilitation, et Cheffe du Département de Médecine interne, Réhabilitation et Gériatrie, Hôpitaux

Universitaires de Genève;

Professeur ordinaire, Faculté de Médecine

Genève

Prof. Dr. med.

Roland von Känel

Titularprofessor Medizinische Fakultät

Universität Bern

Chefarzt Psychosomatische Medizin,

Klinik Barmelweid

Barmelweid

Prof. emer. Dr. med.

Rolf H. Adler

Langjähriger Leiter Psychosomatische Medizin, C.L.

Lory-Haus, Inselspital

Bern/Kehrsatz
Prof. Dr. med.

Friedrich Stiefel

Professeur ordinaire de l'Université de Lausanne, Chef du Service de psychiatrie de liaison du CHUV, Lausanne

Prof. emer. Dr. med.

Claus Buddeberg

Langjähriger Leiter Psychosoziale Medizin, UniversitätsSpital

Zürich

Prof. Dr. phil.

Martin Grosse Holtforth

Assoziierter Professor/Dozent, Institut für Psychologie, Universität Bern

Leiter Forschung Kompetenzbereich Psychosomatische Medizin, C.L. Lory-Haus

Universitätsspital Insel,

Bern

Prof. Dr. med.

Alexander Kiss

Chefarzt

Psychosomatik

Universitätsspital Basel

Basel

Prof. Dr. med

Wolf Langewitz

Stv. Chefarzt

Psychosomatik

Universitätsspital Basel

Basel

Dr. med.

Niklaus Egloff

Leiter Psychosomatische Medizin,

C.L. Lory-Haus

Universitätsspital Insel

Bern
Dr. med. Niklaus Eglo

Leiter Psychosomatisch

Bern 